\title{
Linear Radon-Nikodym Theorems for States on a von Neumann Algebra
}

By

\author{
Hideki KosAKI*
}

\begin{abstract}
Several linear Radon-Nikodym theorems for states on a von Neumann algebra are obtained in the context of a one parameter family of positive cones introduced by $\mathrm{H}$. Araki. Among other results, we determine when a normal state $\phi$ admits a linear Radon-Nikodym derivative with respect to a distinguished normal faithful statc $\dot{\phi}_{0}$ in the sense of Sakai, that is, $\phi=h \dot{\phi}_{0}+\phi_{0} h$ with a positive $h$ in the algebra.
\end{abstract}

\section{§0. Introduction}

We consider a von Neumann algebra on a Hilbert space admitting a cyclic and separating vector. Making use of the associated modular operator, [9], Araki introduced a one parameter family of positive cones. Several RadonNikodym theorems are known in the context of positive cones ([1], [4], [5]) in which "Radon-Nikodym derivatives" reduce to the square roots of measure theoretic Radon-Nikodym derivatives provided that the algebra in question is commutative.

In this paper we obtain three linear Radon-Nikodym theorems (Theorem $1.5,1.6,1.7)$. Our proofs are very constructive so that we obtain explicit expressions of linear Radon-Nikodym derivatives.

Our main tools are relative modular operators and the function $\{\cosh (\pi t)\}^{-1}$ which was used by van Daele to obtain a simple proof of the fundamental theorem of the Tomita-Takesaki theory, [12].

\section{§1. Notations and Main Results}

Let $\mathscr{M}$ be a von Neumann algebra on a Hilbert space $\mathscr{H}$ with a unit cyclic

Communicated by H. Araki, April 30, 1981.

* Department of Mathematics, The University of Kansas, Lawrence, Kansas 66045, U. S. A. 
and separating vector $\xi_{0}$ with the vector state $\phi_{0}=\omega_{\xi_{0}}$, and $\Delta, J$ be the associated modular operator and modular conjugation respectively, [9]. Fixing these throughout, we denote the modular automorphism group $\left\{\operatorname{Ad} \Delta^{i t}\right\}_{t \in \mathbb{R}}$ on $\mathscr{M}$ simply by $\sigma_{t}$. Let $\mathscr{M}_{0}$ be a $\sigma$-weakly dense $*$-subalgebra consisting of every $x \in \mathscr{M}$ such that $t \in \boldsymbol{R} \rightarrow \sigma_{t}(x) \in \mathscr{M}$ extends to an entire function.

The following one parameter family of positive cones was introduced by Araki:

Definition 1.1 ([1]). For each $0 \leqq \alpha \leqq 1 / 2$, we denote the closure of the positive cone $\Delta^{\alpha} \mathscr{M}_{+} \xi_{0}$ in $\mathscr{H}$ by $P^{\alpha}\left(=P_{\phi_{0}}^{\alpha}\right)$. These cones enjoy the following properties:

Proposition 1.2 ([1]). For each $0 \leqq \alpha \leqq 1 / 2$, we have

(i ) $P^{\alpha}=J P^{(1 / 2)-\alpha}=\left(P^{(1 / 2)-\alpha}\right)^{\prime}\left(=\left\{\zeta \in \mathscr{H} ;(\zeta \mid \xi) \geqq 0, \xi \in P^{(1 / 2)-\alpha}\right\}\right.$, the dual cone),

(ii) $P^{\alpha} \cong \mathscr{D}\left(\Delta^{(1 / 2)-2 \alpha}\right)$ and $\Delta^{(1 / 2)-2 \alpha \zeta}=J \zeta, \zeta \in P^{\alpha}$,

(iii) the map: $\xi \in P^{1 / 4} \rightarrow \omega_{\xi} \in \mathscr{M}_{*}^{+}$is bijective.

By (iii) in the above proposition, each $\phi \in \mathscr{M}_{*}^{+}$admits a unique implementing vector in $P^{1 / 4}$, the natural cone, which we will denote by $\xi_{\phi}$, that is, $\phi=\omega_{\xi_{\phi}}$. Then a positive self-adjoint operator $\Delta_{\phi \phi_{0}}$ on $\mathscr{H}$ with a form core $\mathscr{M} \xi_{0}$ satisfying $J \Delta_{\phi \phi_{0}}^{1 / 2} x \xi_{0}=x^{*} \xi_{\phi}, x \in \mathscr{M}$, is known as the relative modular operator (of $\phi$ with respect to $\left.\phi_{0}\right)$. Also, a partial isometry $\Delta_{\phi \phi_{0}}^{i t} \Delta_{\phi_{0}}^{-i t}=\left(D \phi ; D \phi_{0}\right)_{t}, t \in \boldsymbol{R}$, in $\mathscr{M}$ is known as the Radon-Nikodym cocycle (of $\phi$ with respect to $\phi_{0}$ ). (See [3] or $\S 1$ of [5] for full details.)

In the first main theorem (Theorem 1.5), we need the following concept:

Definition 1.3 (One parameter family of orderings, [3]). For $\lambda>0$, we write $\phi \leqq \phi_{0}(\lambda)$ if the map: $t \in \boldsymbol{R} \rightarrow\left(D \phi ; D \phi_{0}\right)_{t}$ extends to a bounded $\sigma$-weakly continuous function on $-\lambda \leqq \operatorname{Im} z \leqq 0$ which is analytic in the interior and $\left\|\left(D \phi ; D \phi_{0}\right)_{-i \lambda}\right\| \leqq 1$. It is well-known that $\phi \leqq \phi_{0}(1 / 2)$ is equivalent to $\phi \leqq \phi_{0}$ in the usual ordering in $\mathscr{M}_{*}$, that is, $\phi(x) \leqq \phi_{0}(x), x \in \mathscr{M}_{+}$.

The next lemma is our main tool in the paper.

Lemma 1.4. Let $f(z)$ be a bounded continuous function on $0 \leqq \operatorname{Re} z \leqq 1$ which is analytic in the interior. We then have

$$
f(1 / 2)=\int_{-\infty}^{\infty}\{f(i t)+f(1+i t)\}\{2 \cosh (\pi t)\}^{-1} d t .
$$

In fact, it is well-known (see p. 208, [8] for example) that the pair $\left(P_{0}\right.$ 
$(\alpha+i \beta, t)=\sin (\pi \alpha) / 2\{\cosh \pi(t-\beta)-\cos \pi \alpha\}, P_{1}(\alpha+i \beta, t)=\sin (\pi \alpha) / 2\{\cosh \pi(t-\beta)$ $+\cos \pi \alpha\}$ ) gives rise to the harmonic measure for the strip $0 \leqq \operatorname{Re} z \leqq 1$. And both of $P_{0}(1 / 2, t)$ and $P_{1}(1 / 2, t)$ are exactly $\{2 \cosh (\pi t)\}^{-1}$. Since we use this result repeatedly, we shall denote the function $\{2 \cosh (\pi t)\}^{-1}$ simply by $F(t)$ throughout the paper.

We now state our three main results. The first one is a slight strengthening of the result in Section 6, [1]. However, more importantly, we obtain the explicit expression of Radon-Nikodym derivatives.

Theorem 1.5. Let $\phi$ be a normal state on $\mathscr{M}$ and $0 \leqq \alpha \leqq 1 / 2$. If $\phi \leqq l \phi_{0}$ $(\operatorname{Max}(\alpha,(1 / 2)-\alpha))$ for some $l>0$, the vector

$$
\zeta_{\alpha}=\int_{-\infty}^{\infty} F(t) \Delta^{(1-2 \alpha) i t}\left(D \phi ; D \phi_{0}\right)_{-i((1 / 2)-\alpha)}^{*} J\left(D \phi ; D \phi_{0}\right)_{-i \alpha}^{*} \xi_{\phi} d t
$$

in $P^{\alpha}$ satisfies $\phi(x)=\left(x \zeta_{\alpha} \mid \xi_{0}\right)+\left(x \xi_{0} \mid \zeta_{\alpha}\right), x \in \mathscr{U}$.

For the special value of $\alpha=0$, the assumption in the theorem is exactly $\phi \leqq l \phi_{0}$ in the usual ordering. Furthermore, $\zeta_{0} \in P^{0}$ is written as $\zeta_{0}=h \xi_{0}$ with $h=\int_{-\infty}^{\infty} F(t) \sigma_{t}\left(\left|\left(D \phi ; D \phi_{0}\right)_{-i / 2}\right|^{2}\right) d t \in \mathscr{L}$ so that we have $\phi=h \phi_{0}+\phi_{0} h$, which is Sakai's linear Radon-Nikodym theorem (Proposition 1, 24, 4, [7]). As the second main result, we prove

Theorem 1.6. Let $\phi$ be a normal state on $\mathscr{M}$. Then $\phi$ admits a (unique) linear Radon-Nikodym derivative $h \in \mathscr{M}_{+}$, that is, $\phi=h \phi_{0}+\phi_{0} h$, if and only if $\tilde{\phi} \leqq l \phi_{0}$ with some $l>0$. Here, $\tilde{\phi} \in \mathscr{M}_{*}^{+}$is defined by

$$
\tilde{\phi}=\int_{-\infty}^{\infty} F(t) \phi \circ \sigma_{t} d t
$$

Furthermore, if this is the case, $h$ is exactly $\left|\left(D \tilde{\phi} ; D \phi_{0}\right)_{-i / 2}\right|^{2}=\left(D \tilde{\phi} ; D \phi_{0}\right)_{-i / 2}^{*}$. $\left(D \tilde{\phi} ; D \phi_{0}\right)_{-i / 2}$. (See also Lemma 4.1.)

In the next result, we further assume that $\phi_{0}$ is periodic in the sense that $\sigma_{T}=$ Id for some $T>0$ ([10]). When $\phi=h \phi_{0}+\phi_{0} h \in \mathscr{M}_{*}^{+}\left(h \in \mathscr{M}_{+}\right)$, for each positive $x$ in the centralizer $\mathscr{M}_{\phi_{0}}([10])$, we estimate

$$
\begin{aligned}
\phi(x) & =\phi_{0}(x h)+\phi_{0}(h x)=2 \phi_{0}\left(x^{1 / 2} h x^{1 / 2}\right) \\
& \leqq 2\|h\| \phi_{0}(x) .
\end{aligned}
$$

Our third result asserts that the converse is also true.

Theorem 1.7. Let $\phi$ be a normal state on $\mathscr{M}$. When the distinguished $\phi_{0}$ is periodic, $\phi$ admits a linear Radon-Nikodym derivative as in the previous 
theorem if and only if $\phi \leqq l \phi_{0}$ on $\mathscr{M}_{\phi_{0}}$, the centralizer of $\mathscr{M}_{\phi_{0}}$, for some $l>0$, that is, $\phi(x) \leqq l \phi_{0}(x)$ for each positive $x \in \mathscr{M}_{\phi_{0}}$.

The rest of the paper is devoted to the proofs of these three theorems. We denote a generic normal state on $\mathscr{M}$ by $\phi$ and the distinguished $\phi_{0}$ is supposed to be periodic only in the proof of the last theorem.

\section{§2. Proof of Theorem $\mathbf{1 . 5}$}

In this section, we prove two lemmas from which Theorem 1.5 follows immediately.

Lemma 2.1. Let $\zeta$ be a vector in $\mathscr{H}$ satisfying

$$
\phi(x)=\left(\Delta^{(1 / 2)-\alpha} x \xi_{0} \mid \zeta\right), \quad x \in \mathscr{M} .
$$

Then $\zeta$ belongs to $P^{\alpha}$. If we set

$$
\eta=\int_{-\infty}^{\infty} F(t) \Delta^{(1-2 \alpha) i t} \zeta d t
$$

then $\eta$ belongs to $P^{\alpha}$ and satisfies

$$
\phi(x)=\left(x \eta \mid \xi_{0}\right)+\left(x \xi_{0} \mid \eta\right), \quad x \in \mathscr{M} .
$$

Proof. The cone $\Delta^{(1 / 2)-\alpha} \mathscr{M}_{+} \xi_{0}$ being dense in $P^{(1 / 2)-\alpha}, \zeta \in P^{\alpha}$ follows from the positivity of $\phi$ and Proposition 1.2, (i). Also, since $P^{\alpha}$ is invariant under $\Delta^{(1-2 \alpha) i t}$ and $F(t)$ is positive for each $-\infty<t<\infty, \eta$ belongs to $P^{\alpha}$ as well.

To prove the final equality, we may and do assume $x \in \mathscr{M}_{0}$. Firstly we observe

$$
\begin{aligned}
\left(x \xi_{0} \mid \eta\right) & =\int_{-\infty}^{\infty} F(t)\left(x \xi_{0} \mid \Delta^{-(1-2 \alpha) i t} \zeta\right) d t \\
& =\int_{-\infty}^{\infty} F(t)\left(\Delta^{(1-2 \alpha) i t} x \xi_{0} \mid \zeta\right) d t \\
& =\int_{-\infty}^{\infty} F(t)\left(\Delta^{(1 / 2)-\alpha} \sigma_{(1-2 \alpha) t+i((1 / 2)-\alpha)}(x) \xi_{0} \mid \zeta\right) d t \\
& =\int_{-\infty}^{\infty} F(t) \phi\left(\sigma_{(1-2 \alpha) t+i((1 / 2)-\alpha)}(x)\right) d t .
\end{aligned}
$$

Secondly, by Proposition 1.2, (ii), we observe

$$
\begin{aligned}
\left(x \eta \mid \xi_{0}\right) & =\left(J \Delta^{(1 / 2)-2 \alpha} \eta \mid x^{*} \xi_{0}\right)=\left(J \Delta^{(1 / 2)-2 \alpha} \eta \mid J \Delta^{1 / 2} x \xi_{0}\right) \\
& =\left(\Delta^{1 / 2} x \xi_{0} \mid \Delta^{(1 / 2)-2 \alpha} \eta\right)=\left(\Delta^{1-2 \alpha} x \xi_{0} \mid \eta\right) \\
& =\int_{-\infty}^{\infty} F(t)\left(\Delta^{1-2 \alpha} x \xi_{0} \mid \Delta^{-(1-2 \alpha) i t}\right) d t
\end{aligned}
$$




$$
\begin{aligned}
& =\int_{-\infty}^{\infty} F(t)\left(\Delta^{1-2 \alpha+(1-2 \alpha) i t} x \xi_{0} \mid \zeta\right) d t \\
& =\int_{-\infty}^{\infty} F(t)\left(\Delta^{(1 / 2)-\alpha} \sigma_{(1-2 \alpha) t-i((1 / 2)-\alpha)}(x) \xi_{0} \mid \zeta\right) d t \\
& =\int_{-\infty}^{\infty} F(t) \phi\left(\sigma_{(1-2 \alpha) t-i((1 / 2)-\alpha)}(x)\right) d t .
\end{aligned}
$$

Hence, Lemma 1.4 applied to $f(z)=\phi\left(\sigma_{w}(x)\right), w=-i(1-2 \alpha)(z-(1 / 2))$, yields:

$$
\left(x \xi_{0} \mid \eta\right)+\left(x \eta \mid \xi_{0}\right)=\phi\left(\sigma_{0}(x)\right)=\phi(x) .
$$

Q.E.D.

Lemma 2.2. If $\phi \leqq l \phi_{0}(\operatorname{Max}(\alpha,(1 / 2)-\alpha))$, then we have

$$
\phi(x)=\left(\Delta^{(1 / 2)-\alpha} x \xi_{0} \mid \zeta\right), \quad x \in \mathscr{M}
$$

with

$$
\zeta=\left(D \phi ; D \phi_{0}\right)_{-i((1 / 2)-\alpha)}^{*} J\left(D \phi ; D \phi_{0}\right)_{-i \alpha}^{*} \xi_{\phi} .
$$

Proof. We simply compute

$$
\begin{aligned}
\left(\Delta^{(1 / 2)-\alpha} x \xi_{0} \mid \zeta\right) & =\left(\left(D \phi ; D \phi_{0}\right)_{-i((1 / 2)-\alpha)} \Delta^{(1 / 2)-\alpha} x \xi_{0} \mid J\left(D \phi ; D \phi_{0}\right)_{-i \alpha} \xi_{\phi}\right) \\
& =\left(\left(D \phi ; D \phi_{0}\right)_{-i((1 / 2)-\alpha)} \Delta^{(1 / 2)-\alpha} x \xi_{0} \mid \Delta_{\phi \phi_{0}}^{1 / 2}\left(D \phi ; D \phi_{0}\right)_{-i \alpha} \xi_{0}\right) .
\end{aligned}
$$

Using the uniqueness of analytic continuation, we can easily prove

$$
\begin{aligned}
& \left(D \phi ; D \phi_{0}\right)_{-i((1 / 2)-\alpha)} \Delta^{(1 / 2)-\alpha} x \xi_{0}=\Delta_{\phi \phi_{0}}^{(1 / 2)-\alpha} x \xi_{0}, \\
& \left(D \phi ; D \phi_{0}\right)_{-i \alpha} \xi_{0}=\Delta_{\phi \phi_{0}}^{\alpha} \xi_{0} .
\end{aligned}
$$

Since $\left(D \phi ; D \phi_{0}\right)_{-i \alpha} \xi_{0}$ belongs to $\mathscr{M} \xi_{0} \subseteq \mathscr{D}\left(\Delta_{\phi \phi_{0}}^{1 / 2}\right)$, we have $\xi_{0} \in \mathscr{D}\left(\Delta_{\phi \phi_{0}}^{(1 / 2)+\alpha}\right)$ and

$$
\begin{aligned}
\left(\Delta^{(1 / 2)-\alpha} x \xi_{0} \mid \zeta\right) & =\left(\Delta_{\phi \phi_{0}}^{(1 / 2)-\alpha} x \xi_{0} \mid \Delta_{\phi \phi_{0}}^{(1 / 2)+\alpha} \xi_{0}\right) \\
& =\left(\Delta_{\phi \phi_{0}}^{1 / 2} x \xi_{0} \mid \Delta_{\phi \phi_{0}}^{1 / 2} \xi_{0}\right)=\left(J x^{*} \xi_{\phi} \mid \xi_{\phi}\right) \\
& =\left(\xi_{\phi} \mid x^{*} \xi_{\phi}\right)=\phi(x) .
\end{aligned}
$$

\section{§3. Proof of Theorem 1.6}

The proof of Theorem 1.6 is divided into three lemmas.

Lemma 3.1. Assume that $\phi=h \phi_{0}+\phi_{0} h \in \mathscr{M}_{*}^{+}$with a positive $h \in \mathscr{M}$. Then for each $x \in \mathscr{M}$ we have

$$
\begin{aligned}
\phi_{0}\left(x \sigma_{-i / 2}(h)\right) & =\int_{-\infty}^{\infty} F(t) \phi\left(\sigma_{-t}(x)\right) d t \\
& =\int_{-\infty}^{\infty} F(t) \phi\left(\sigma_{t}(x)\right) d t
\end{aligned}
$$

Here, the left hand side makes sense due to the K.M.S. condition, [9]. 
Proof. By the invariance $\phi_{0} \circ \sigma_{t}=\phi_{0}$, we compute

$$
\begin{aligned}
\int_{-\infty}^{\infty} F(t) \phi\left(\sigma_{-t}(x)\right) d t & =\int_{-\infty}^{\infty}\left\{\phi_{0}\left(\sigma_{-t}(x) h\right)+\phi_{0}\left(h \sigma_{-t}(x)\right)\right\} F(t) d t \\
& =\int_{-\infty}^{\infty}\left\{\phi_{0}\left(x \sigma_{t}(h)\right)+\phi_{0}\left(\sigma_{t}(h) x\right)\right\} F(t) d t
\end{aligned}
$$

Thus, the result follows from Lemma 1.4 and the fact that $\phi_{0}\left(x \sigma_{z}(h)\right)$ is bounded analytic on $-1 \leqq \operatorname{Im} z \leqq 0$ and $\phi_{0}\left(x \sigma_{-i+t}(h)\right)=\phi_{0}\left(\sigma_{t}(h) x\right)$ (the K.M.S. condition).

Q.E.D.

Lemma 3.2. For $x, h \in \mathscr{M}_{+}$, we have

$$
0 \leqq \phi_{0}\left(x \sigma_{-i / 2}(h)\right) \leqq\|h\| \phi_{0}(x) .
$$

Proof. We notice that

$$
\begin{aligned}
\phi_{0}\left(x \sigma_{-i / 2}(h)\right) & =\left(x \Delta^{1 / 2} h \xi_{0} \mid \xi_{0}\right) \\
& =\left(x J h J \xi_{0} \mid \xi_{0}\right)
\end{aligned}
$$

due to the positivity of $h$. Since $0 \leqq J h J \leqq\|h\|$ and they commute with $x \in \mathscr{M}_{+}$, we have

$$
0 \leqq\left(x J h J \xi_{0} \mid \xi_{0}\right) \leqq\|h\|\left(x \xi_{0} \mid \xi_{0}\right)=\|h\| \phi_{0}(x) . \quad \text { Q.E.D. }
$$

Lemma 3.3. When

$$
\tilde{\phi}=\int_{-\infty}^{\infty} F(t) \phi \circ \sigma_{t} d t \leqq l \phi_{0},
$$

we have $\phi=h \phi_{0}+\phi_{0} h$ with $h=\left|\left(D \tilde{\phi} ; D \phi_{0}\right)_{-i / 2}\right|^{2} \in \mathscr{M}_{+}$.

Proof. To prove $\phi(x)=\phi_{0}(x h)+\phi_{0}(h x)$, we may and do assume that $x \in \mathscr{M}_{0}$. At first we notice that

$$
\begin{aligned}
\left(D \tilde{\phi} ; D \phi_{0}\right)_{-i / 2} \xi_{0} & =\xi_{\tilde{\phi}}, \\
\left(D \tilde{\phi} ; D \phi_{0}\right)_{-i / 2} y \xi_{0} & =\left(D \tilde{\phi} ; D \phi_{0}\right)_{-i / 2} J \Delta^{1 / 2} y^{*} \xi_{0} \\
& =\left(D \tilde{\phi} ; D \phi_{0}\right)_{-i / 2} J \sigma_{-i / 2}\left(y^{*}\right) J \xi_{0} \\
& =J \sigma_{-i / 2}\left(y^{*}\right) J\left(D \tilde{\phi} ; D \phi_{0}\right)_{-i / 2} \xi_{0} \\
& =J \sigma_{-i / 2}\left(y^{*}\right) \xi_{\tilde{\phi}} \quad\left(y \in \mathscr{M}_{0}\right) .
\end{aligned}
$$

By using these facts, it is easily shown that

$$
\begin{aligned}
\phi_{0}(h x)+\phi_{0}(x h) & =\tilde{\phi}\left(\sigma_{i / 2}(x)+\sigma_{-i / 2}(x)\right) \\
& =\int_{-\infty}^{\infty} F(t)\left\{\phi\left(\sigma_{i / 2+t}(x)\right)+\phi\left(\sigma_{-i / 2+t}(x)\right)\right\} d t
\end{aligned}
$$

Thus, the result follows from Lemma 1.4 applied to the function 


$$
f(z)=\phi\left(\sigma_{w}(x)\right), \quad w=-i\left(z-2^{-1}\right) . \quad \text { Q.E. D. }
$$

\section{§4. Proof of Theorem 1.7}

The next lemma is obtained in [6] in a slightly different set up. However, for the sake of completeness, we present its proof in our context.

Lemma 4.1. We have

$$
\tilde{\phi}=\int_{-\infty}^{\infty} F(t) \phi \circ \sigma_{t} d t \leqq l \phi_{0}
$$

if and only if for each $\alpha>0$ (hence all $\alpha$ ) there exists a positive $c=c_{\alpha}$ such that

$$
\int_{-\alpha}^{\alpha} \phi \circ \sigma_{t} d t \leqq c \phi_{0}
$$

Proof. The "only if" part is trivial since $F(t)$ is a strictly positive even function and monotone decreasing on $[0, \infty)$. To show the "if" part, we assume that

$$
\int_{-\alpha}^{\alpha} \phi \circ \sigma_{t} d t \leqq c \phi_{0}
$$

We compute that

$$
\begin{aligned}
\int_{-\infty}^{\infty} F(t) \phi \circ \sigma_{t} d t & =\sum_{n=-\infty}^{\infty} \int_{(2 n-1) \alpha}^{(2 n+1) \alpha} F(t) \phi \circ \sigma_{t} d t \\
& =\sum_{n=-\infty}^{\infty} \int_{-\alpha}^{\alpha} F(t+2 n \alpha) \phi \circ \sigma_{t+2 n \alpha} d t \\
& \leqq \sum_{n=-\infty}^{\infty} c\{\exp (\pi(2|n|+1) \alpha)\}^{-1} \phi_{0} \circ \sigma_{2 n \alpha} \\
& =\left(\sum_{n=-\infty}^{\infty} c\{\exp (\pi(2|n|+1) \alpha)\}^{-1}\right) \phi_{0} .
\end{aligned}
$$

Q.E.D.

Proof of the theorem. Let $T>0$ be the period of $\sigma_{t}$. It is easy to observe that

$$
\int_{-T}^{T} \sigma_{t} d t=2 T \varepsilon
$$

where $\varepsilon$ is a normal projection of norm 1 from $\mathscr{M}$ onto the centralizer $\mathscr{M}_{\phi_{0}}$ satisfying $\phi_{0^{\circ} \varepsilon}=\phi_{0}$. (See [2], [10], [11].) By the previous lemma and Theorem 1.6 , we know that $\phi=h \phi_{0}+\phi_{0} h$ if and only if $\phi \circ \varepsilon \leqq l^{\prime} \phi_{0}=l^{\prime} \phi_{0} \circ \varepsilon$, which is equivalent to $\phi \leqq l^{\prime} \phi_{0}$ on $\mathscr{M}_{\phi_{0}}$.

Q.E.D. 


\section{References}

[1] Araki, H., Some properties of modular conjugation operator of von Neumann algebras and a non-commutative Radon-Nikodym theorem with a chain rule, Pacific $J$. of Math., 50 (1974), 309-354.

[2] Combes, F. and Delaroche, C., Groupe modulaire d'une espérance conditionelle dans une algèbre de von Neumann, Bull. Soc. Math. France, 103 (1975), 385-426.

[ 3 ] Connes, A. and Takesaki, M., The flow of weights on factors of type III, Tohoku Math. J., 29 (1977), 473-575.

[4] Kosaki, H., Positive cones associated with a von Neumann algebra, Math. Scand, 47 (1980), 295-307.

[5] —, Positive cones and $L^{p}$-spaces associated with a von Neumann algebra, J. Operator Theory, 6 (1981), 13-23.

[6] Pedersen, G., On the operator equation $H T+T H=2 K$, preprint.

[7] Sakai, S., $C^{*}$-algebras and $W^{*}$-algebras, Springer Verlag, Berlin-Heidelberg-New York, 1971.

[ 8 ] Stein E. and Weiss G., Introduction to Fourier analysis on Euclidean spaces, Princeton Univ. Press, 1971.

[9] Takesaki, M., Tomita's theory of modular Hilbert algebras and its applications, Lecture Notes in Math., 128, Springer Verlag, Berlin-Heidelberg-New York, 1970.

[10] — The structure of a von Neumann algebra with a homogeneous periodic state, Acta Math., 131 (1973), 79-121.

[11] — Conditional expectations in von Neumann algebras, J. Funct. Anal., 9 (1972), 306-321.

[12] van Daele, A., A new approach to the Tomita-Takesaki theory for von Neumann algebras with a cyclic and separating vector, J. Funct. Anal., 15 (1974), 278-393. 